\title{
Efficacy evaluation of reteplase in a novel canine acute pulmonary thromboembolism model developed by minimally invasive surgery and digital subtraction angiography
}

This article was published in the following Dove Press journal:

Drug Design, Development and Therapy

Yinbing Zhang, ${ }^{1,2}$ Haifeng Liu, ${ }^{3}$ Yingqian Zhang, ${ }^{4}$ Qiong Wu, ${ }^{1}$ Yanyan Zhang, ${ }^{1,2}$ Jie Zhang, ${ }^{1,2}$ Xiangshan Zhou, ${ }^{3} \mathrm{He}$ Jiao, ${ }^{5}$ Feng Fan, ${ }^{6}$ Qi Xue, ${ }^{7}$ Xin Wang, ${ }^{1,2}$ Zhihui Zhong, ${ }^{1,2}$

'Laboratory of Nonhuman Primate Disease Modeling Research, State Key Laboratory of Biotherapy, West China Hospital, Sichuan University, Chengdu, China; ${ }^{2}$ Sichuan Kangcheng Biomed Co. Ltd., Chengdu, China; ${ }^{3}$ Angde Biotech Pharmaceutical Co., Ltd., Liaocheng, China; ${ }^{4}$ Department of Physiology, Southwest Medical University, Luzhou, China; ${ }^{5}$ Department of Interventional therapy, West China Hospital, Sichuan University, Chengdu, China; ${ }^{6}$ Department of Neurointervention, The First Affiliated Hospital of Zhengzhou University, Zhengzhou, China; ${ }^{7}$ Food and Drug Administration of Shibei District Government, Qingdao, China

Correspondence: Xin Wang Sichuan Kangcheng Biomed Co., Ltd., 28 Gaopeng Avenue, Chengdu,

Sichuan, 6I004I, China

Tel +862883326312

Fax +86 2883326313

Email xin.wang@hcbiomed.com

Zhihui Zhong

Laboratory of Nonhuman Primate Disease Modeling Research, State Key Laboratory of Biotherapy, West China Hospital, Sichuan University, 28 Gaopeng Avenue, Chengdu,

Sichuan, 61004I, China

Tel +8628833263I2

Fax +862883326313

Email zhongzhihui@scu.edu.cn
Purpose: In order to evaluate the thrombolytic effects of reteplase in pulmonary thromboembolism (PTE), we developed a novel canine PTE model. The efficacy of reteplase against PTE in comparison to alteplase was clarified for the first time, and this PTE model could be further applied to studies of novel thrombolytic therapies.

Patients and methods: Twenty-four dogs were divided into four groups: sham operation, vehicle, alteplase, and reteplase. Autologous thrombi/saline were injected into the pulmonary artery, and thrombolytic agents were administrated. Thrombus formation and dissolution were monitored by real-time digital subtraction angiography (DSA), and pulmonary pressures were measured simultaneously. Blood coagulation, blood gas, hematology, and histopathologic examinations were used as subsidiary methods.

Results: The canine PTE model was established with a significant decrease of blood flow and $\sim 75 \%$ blocking area. Administration of reteplase $(0.6 \mathrm{mg} / \mathrm{kg})$ resulted in effective thrombus dissolution with a recovery of over $80 \%$ blood flow, as effective as alteplase $(1.6 \mathrm{mg} / \mathrm{kg})$. Correspondingly, the elevated pulmonary systolic, diastolic, and mean arterial pressures declined to the normal level. Blood coagulation was changed by reteplase, with a dramatic elongation of prothrombin time, activated partial thromboplastin time, and thrombin time, even longer than alteplase. In contrast to the vehicle group, no obvious pathological changes were found in the two thrombolytic groups. Hematological, blood biochemical, and blood gas results also indicated that reteplase had no adverse reactions in this PTE model.

Conclusion: Reteplase proved to be an effective and safe therapy for PTE for the first time, and a small dosage of reteplase exerted an efficacy comparable to the routine dosage of alteplase. Our findings indicated the potential of reteplase as clinical treatment against PTE. This technically innovative, stability- and validity-proved canine PTE model developed by minimally invasive surgery and DSA resembled major clinical features. This may further facilitate our understanding of thrombotic disorders and development of prophylactic and therapeutic approaches.

Keywords: reteplase, canine pulmonary thromboembolism model, thrombolytic therapy

\section{Introduction}

With a high morbidity and mortality, pulmonary thromboembolism (PTE) has become the third most common cause of death among hospitalized patients following stroke and myocardial infarction worldwide. ${ }^{1-3}$ With $\sim 600,000$ cases annually and 100,000-180,000 deaths related to PTE all over the world, sub-massive and massive pulmonary embolism have a high rate of mortality from $28 \%$ up to $68 \% .^{4,5}$ 
The incidence rate of PTE was as high as 112 cases per 100,000 person-years in USA alone. ${ }^{6}$ The 2011 guidelines of the American Heart Association and the guidelines of the American College of Chest Physicians recommended that patients with massive pulmonary embolism, evidence of hemodynamic compromise, and acceptable bleeding risk should receive thrombolytic treatment. ${ }^{7,8}$ Therefore, development of efficient and effective therapies against PTE is of great clinical significance. Thrombolytic regimens, such as alteplase, streptokinase, and urokinase, were usually recommended clinically as treatments against massive PTE and sub-massive PTE. Clinical treatment on time could largely reduce the mortality rate of PTE from $\sim 30 \%$ to $4 \%-8 \%{ }^{9-12}$

Recombinant human tissue-type plasminogen activator derivative (reteplase), as a third generation of thrombolytic agents, is used as thrombolytic treatment in patients with acute ST-segment elevation myocardial infarction (STEMI) caused by coronary artery infarction. ${ }^{13}$ ST-segment refers to the flat section of an electrocardiography, and when a person has a heart attack, this segment will become abnormally elevated. Reteplase, a $39.6 \mathrm{kD}$ recombinant protein with 355 amino acids, contains the kringle 2 and protease domains of the native tissue plasminogen activator (t-PA). Due to the lack of kringle 1 and fibronectin finger domains, and the epidermal growth factor binding domain, the binding capability of reteplase to the hepatocyte receptors was reduced. Therefore, reteplase was less recognized and degraded by hepatocytes, which elongated its half-life by 3-5 times. Reteplase could catalyze the inactive proenzyme plasminogen into the active protease plasmin, which subsequently degrades fibrin in the thrombus and leads to consequent thrombolysis. Reteplase was characterized with a higher thrombolytic potential than alteplase in the pharmacokinetic-pharmacodynamics perspective. ${ }^{14}$ In a canine model of coronary artery thrombosis, reteplase produced more beneficial reperfusion effects than alteplase, streptokinase, and urokinase. ${ }^{15} \mathrm{Up}$ to now, reteplase has shown excellent thrombolytic effect in patients with myocardial infarction; however, its efficacy as a treatment for PTE is yet to be established.

During the development of novel anti-PTE drugs, preclinical studies were of great significance. The establishment of a proper PTE animal model with analogous clinical characteristics became more and more critical for screening and efficacy evaluation of novel anti-PTE drugs. Inferior vena cava (IVC) ligation rodent models were widely used in the study of deep venous thrombosis (DVT), with obvious disadvantages though. The poor blood flow inhibited the maximal effects of systemic therapeutic agents on the thrombi, and hemodynamic parameters were not easy to monitor. ${ }^{16}$ Currently, large animal models of PTE involving canines and pigs established by injecting thrombi or foreign bodies into the main pulmonary vessels exhibited similar disease symptoms compared to clinical patients. However, due to the effects of the fibrinolytic system, unstable shapes of blood clots might flow freely into other pulmonary arteries, and it was difficult to control the extent and persistence of these occlusions. ${ }^{17-19}$

Herein, in order to accurately evaluate the thrombolytic effects of reteplase in PTE, we developed a novel Beagle dog PTE model with autologous thrombus-induced blockage in the pulmonary artery through minimally invasive surgery. The reliability and applicability of this system were verified by the well-acknowledged thrombolytic drug, alteplase, which we used as a positive control. The thrombolytic capability of reteplase was systematically verified in a "what you see is what you get" way in this canine PTE model, by means of pulmonary digital subtraction angiography (DSA), the classic clinically diagnostic method of PTE. Hemodynamic, blood coagulation, blood gas, hematological, blood biochemical indexes, necropsy, and histopathologic examinations were used as subsidiary assessing indicators. Here, we developed a novel accurate and effective canine PTE model, which resembled the pathogenic and pathophysiological changes during disease progression. Reteplase, a clinical treatment for STEMI, proved to be an effective and safe therapy for PTE for the first time.

\section{Materials and methods}

\section{Animals}

Beagle dogs (12 males and 12 females), weighing 8.0-12.0 kg and aged 7-12 months, were provided by Guangzhou Medical Research Institute Co, Ltd, China. At least 2 weeks were given to the animals to adapt to the laboratory environment (temperature: $18^{\circ} \mathrm{C}-25^{\circ} \mathrm{C}$, relative humidity: $40 \%-70 \%$ ), and healthy ones (nonpregnant if female) were chosen for the experiment. All experimental procedures were conducted in accordance with the guiding principles for the care and use of animals in research, approved by Sichuan University, China. The experiments were approved by the Animal Care and Ethics Committee of the West China Hospital, Sichuan University (Chengdu, China).

\section{Thrombolytic agents}

Reteplase (lot: 201511005, 39.6 kD, fibrin-specific activity $\geq 9.0 \times 10^{4} \mathrm{IU} / \mathrm{mg}$ ) was provided by Angde Biotech Pharmaceutical Co, Ltd, Shandong, China. Alteplase 
(lot: 406396) was purchased from Boehringer Ingelheim, Ingelheim, Germany.

\section{Groups and drug administration}

Twenty-four dogs were randomly divided into four groups by weight (six dogs per group): the sham operation group, the vehicle group, the alteplase group, and the reteplase group. After thrombi in the pulmonary artery stabilized for 1 hour, dogs in the reteplase group were administrated bolus injection once $(0.6 \mathrm{mg} / \mathrm{kg})$ within 2 minutes, and dogs in the alteplase group were treated with alteplase $(1.6 \mathrm{mg} / \mathrm{kg}, 6 \mathrm{~mL} / \mathrm{kg})$ by intravenous infusion for 30 minutes. Animals in the sham operation group and the vehicle group underwent the same procedure with intravenous infusion of physiological saline $(6 \mathrm{~mL} / \mathrm{kg})$.

\section{Establishment of the PTE model}

Blood $(5 \mathrm{~mL} / \mathrm{kg})$ was extracted from the extremity vein of the experimental animals, and injected into the sterile plastic capillary. Thrombus with an approximate length of $5 \mathrm{~cm}$, diameter of $1 \mathrm{~mm}$ was generated, kept in a $37^{\circ} \mathrm{C}$ water bath for 30 minutes after being sealed, followed by $2^{\circ} \mathrm{C}-8^{\circ} \mathrm{C}$ overnight, and sucked into a syringe as backup.

After fasting for 12-16 hours, all dogs were anesthetized by vein injection of pentobarbital sodium $(30 \mathrm{mg} / \mathrm{kg}$, lot: 020M2298V; Sigma-Aldrich Co, St Louis, MO, USA), and the intravenous line was established in the animal's left arm. Peripheral arterial pressures were monitored by a pressure sensor placed in the femoral artery via the indwelling needle after anesthesia. Femoral vein puncture was performed by the Seldinger method. Upon successful puncture, a catheter sheath was placed, and a 5-F guiding catheter was inserted into the left pulmonary artery with the guidance of the super hydrophilic guide wire. After confirmation of the proper positioning of the 5-F guiding catheter, the pressure sensor was connected and used to monitor the pulmonary arterial pressures. Contrast medium was injected through the 5-F catheter sheath before model establishment for angiography. Afterward, the pre-prepared thrombi were injected into the left pulmonary main artery or its major branch through the 5-F guiding catheter at a volume of $3 \mathrm{~mL}$ each time under DSA. Three clots of thrombus (a total volume of $9 \mathrm{~mL}$ per dog) were injected into each dog in the vehicle group, the alteplase group, and the reteplase group. The same amount of saline was injected into the sham operation group. The contrast medium was injected through the 5-F catheter sheath into the right femoral vein, so as to enable angiography of the embolic sites and to ensure real-time blocking of the pulmonary arteries. Results of the angiography were graded based on the Miller standard, ${ }^{20}$ and level 1 (moderately reduced blood flow volume, $<50 \%$ ) was considered as a successful PTE model.

\section{Pulmonary angiography analysis}

The contrast medium was injected through the 5-F catheter sheath into the femoral vein of the animal, immediately before, right after, and 1 hour after thrombus injection and immediately after, 60, and 120 minutes after drug administration. Pulmonary angiography of the embolic sites was carried out under DSA (Allura Xper FD20; Philips, Amsterdam, the Netherlands) and graded by the Miller standard.

\section{Hemodynamic measurements}

The pulmonary arterial systolic pressure, pulmonary arterial diastolic pressure, and pulmonary mean arterial pressure (MAP) were monitored (Multichannel Physiology Recorder, MP150; Biopac, Goleta, CA, USA) immediately before, right after, and 1 hour after thrombus injection and immediately, 60 , and 120 minutes after drug administration.

\section{Analysis of blood gas}

Approximately $1 \mathrm{~mL}$ of peripheral venous blood was collected from the animal's femoral artery via the indwelling needle, immediately before, after, and 1 hour after thrombus injection and immediately, 60, and 120 minutes after drug administration. The blood sample (non-anticoagulant) was analyzed by an i-STAT 1 blood gas analyzer (Abbott Laboratories, Abbott Park, IL, USA).

\section{Hematology and blood chemistry examination}

Blood was collected from the cephalic vein in the dog's forelimb before thrombus injection, 1 hour after thrombus injection, 1 hour after drug administration, and 6 days later. Blood sample was anticoagulated with EDTA for classification and examination of blood cells. Hematological test parameters included red blood cell, hemoglobin, hematocrit, mean corpuscular volume, mean corpuscular hemoglobin, mean corpuscular hemoglobin concentration, reticulocyte, white blood cell, neutrophil, lymphocyte, monocyte, eosinophils, basophilic granulocyte, and platelet counts (automatic blood analyzer, ADVIA2120; Siemens, Munich, Germany). Blood samples were anticoagulated with sodium citrate, and plasma was separated for the examination of blood coagulation indexes. The examined blood coagulation indexes included prothrombin time $(\mathrm{PT})$, activated partial thromboplastin time 
(APTT), thrombin time (TT), and fibrinogen (FIB) (automatic coagulation analyzer, CA-7000; Sysmex Corporation, Kobe, Japan). Serum was separated for examination of blood biochemical indexes, such as aspartate amino transferase, alanine aminotransferase, creatine phosphokinase, alkaline phosphatase, urea, L- $\gamma$-glutamyl transpeptidase, total protein, albumin, albumin-globulin ratio, blood glucose, creatinine, total bilirubin, total cholesterol, triglycerides, total serum calcium, and concentrations of $\mathrm{Na}^{+}, \mathrm{K}^{+}, \mathrm{Cl}^{-}$(Cobas C501; Hoffman-La Roche Ltd, Basel, Switzerland).

\section{Necropsy and histopathologic examination}

Surviving animals were euthanized and autopsy was conducted at day 7 of the experiment. All animals were fasted at least 12 hours before the dissection. Animals to be euthanized were first anesthetized with pentobarbital $(30 \mathrm{mg} / \mathrm{kg}$, intravenous injection). After a thorough observation of the appearance, animals were exsanguinated via the femoral artery for euthanasia, followed by necropsy. Presence of bleeding in all organs was observed. Pathological specimens were made and fixed by $10 \%$ formaldehyde solution. The pulmonary lobe with thrombus injected was taken and paraffin-embedded. A 4- $\mu \mathrm{m}$ thick slice was generated and stained with Prussian blue. Morphological changes in the vascular intima of the pulmonary arteries, lung interstitium, and alveoli were observed under a microscope.

\section{Statistical analysis}

SPSS program (version 22.0; IBM Corporation, Armonk, NY, USA) was used for all data analysis. Due to the small sample size in each group $(n=6)$, and according to the Shapiro-Wilk test and Q-Q plots, the data did not fully conform to a normal distribution. Pairwise comparisons in the parameters were performed by nonparametric tests (Kruskal-Wallis) followed by Dunn's post hoc test to determine the differences. Measurement data were presented as median (range: $\min -\max$ ). A $P$-value $<0.05$ was considered statistically significant.

\section{Results}

\section{Establishment of the novel canine PTE model}

After injection of the pre-prepared quantitative autologous thrombi or same amount of saline into the secondary branch of the dog's pulmonary artery by using the minimally invasive guiding catheter, pulmonary angiography was carried out under DSA at different time points as indicated to visualize the embolic sites (Figure 1A, Videos $\mathrm{S} 1-\underline{\mathrm{S} 4}$ ). Comparison of angiography results before and after thrombus injection in both the vehicle and treatment groups suggested successful establishment of embolisms, with a significant decrease of blood flow volume in the pulmonary artery, which stabilized till 60 minutes after thrombus injection, with $\sim 75 \%$ blocking area in the median (Figure 1B). In contrast, dogs in the sham operation group did not show noticeable change of blood flow volume after injection of the same volume of saline (Figure 1A). This PTE model was graded to have high sensitivity by the Miller standard, suggesting its suitability and capability for the evaluation of thrombolytic drugs.

\section{Thrombolytic effects evaluation of reteplase in the PTE model}

Real-time thrombolytic effects of reteplase were captured by angiography under DSA. Blocking area of pulmonary embolism was calculated as (pixel of pulmonary artery before thrombus injection - pixel of pulmonary artery after thrombus injection or drug administration)/pixel of pulmonary artery before thrombus injection $\times 100$ (Figure 1B). As stated above, embolism was successfully established and sustained for $>1$ hour in the vehicle, alteplase, and reteplase group, with $\sim 75 \%$ blocking area. Partial recovery of blood flow, with blocking area decreased from $79.53 \%$ (74.35\%-87.39\%) to $59.54 \%$ (37.76\%-77.49\%), was achieved immediately after a single intravenous bolus injection of reteplase $(0.6 \mathrm{mg} / \mathrm{kg})$. Continuous large recovery of blood flow was observed 60 and 120 minutes after the bolus injection, and the blocking area was decreased to $32.02 \%(20.10 \%-46.57 \%)$ and $16.79 \%$ (11.47\%-34.08\%), respectively. Compared to the vehicle group, with $68.42 \%$ (57.29\%-78.57\%) blocking area 2 hours after drug administration, the vessel blocking area in the reteplase group was significantly reduced $(P=0.028, \mathrm{n}=6)$.

In contrast, alteplase $(1.6 \mathrm{mg} / \mathrm{kg})$ induced a similar level of recanalization as reteplase $(0.6 \mathrm{mg} / \mathrm{kg})$ in this canine PTE study $(P=1, P=1,60$ and 120 minutes after drug administration, respectively, $n=6$ ). The median blocking area of pulmonary artery in the alteplase group was reduced to $31.55 \%$ (11.21\%-38.29\%) and 12.70\% (6.96\%-36.45\%), 60 and 120 minutes after drug administration, respectively, significantly different from the original $79.33 \%(69.75 \%-83.82 \%)$ upon thrombus injection $(P=0.007$ and $P=0.004$, respectively, $\mathrm{n}=6)$. In contrast, $68.42 \%(57.29 \%-78.57 \%)$ blocking area was sustained in the vehicle group 120 minutes after drug (saline) administration, suggesting sustainability of the embolism and verifying the thrombolytic effects of both reteplase and alteplase (Figure 1B, Videos S1- $\underline{\mathrm{S} 4}$ ). 


\section{A}
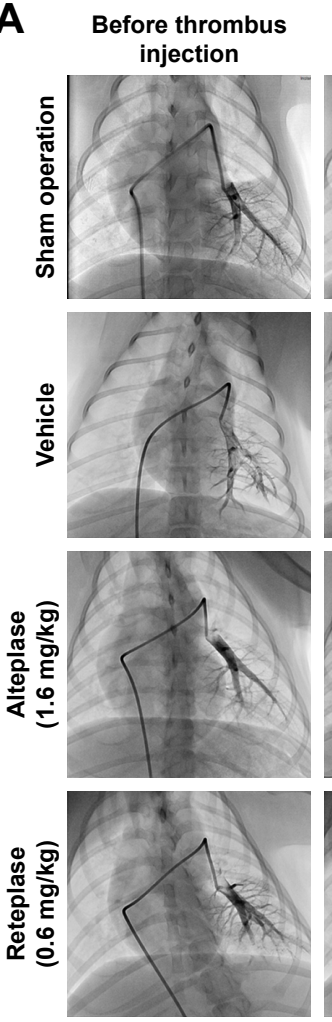
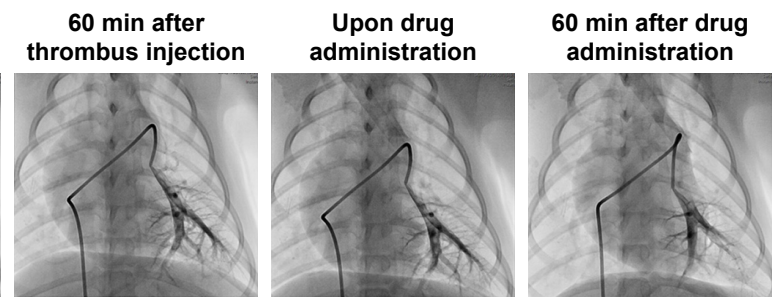

120 min after drug
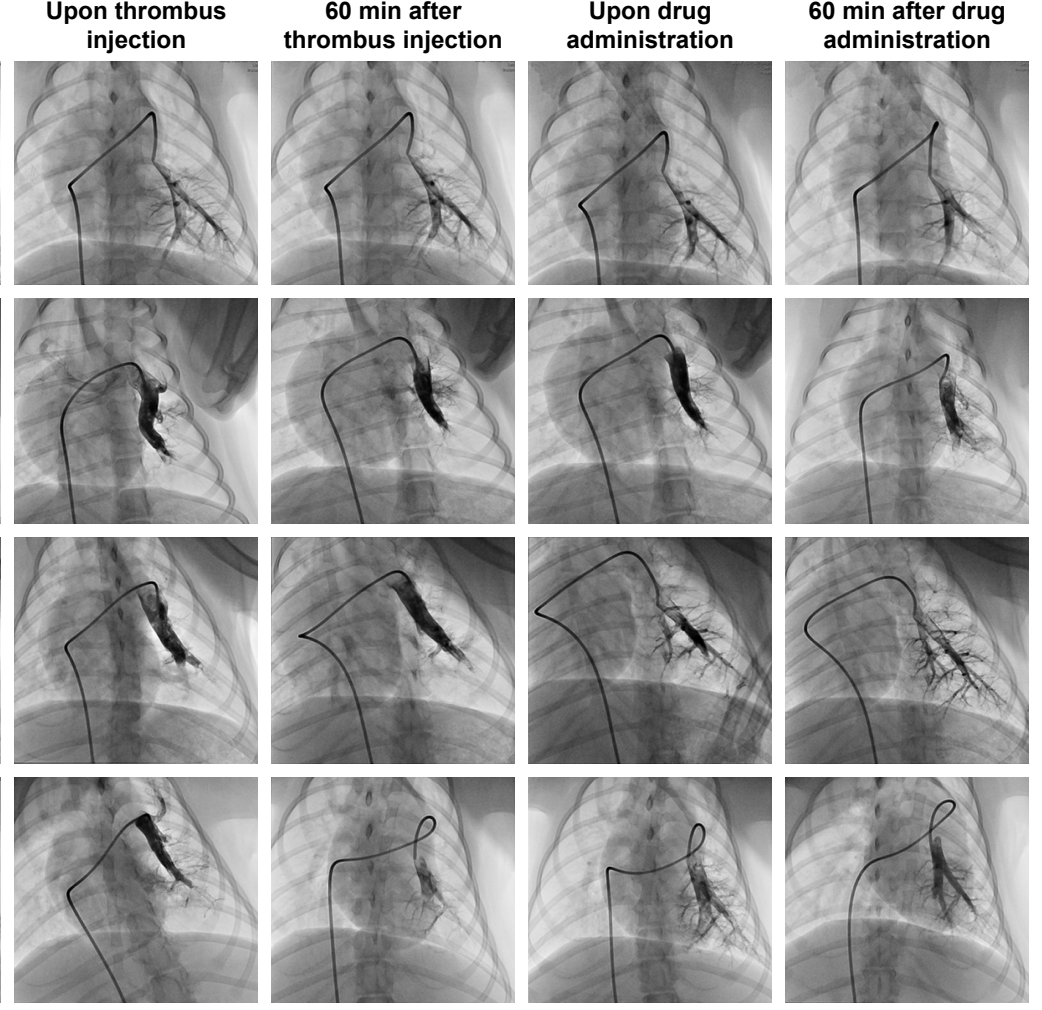
administration

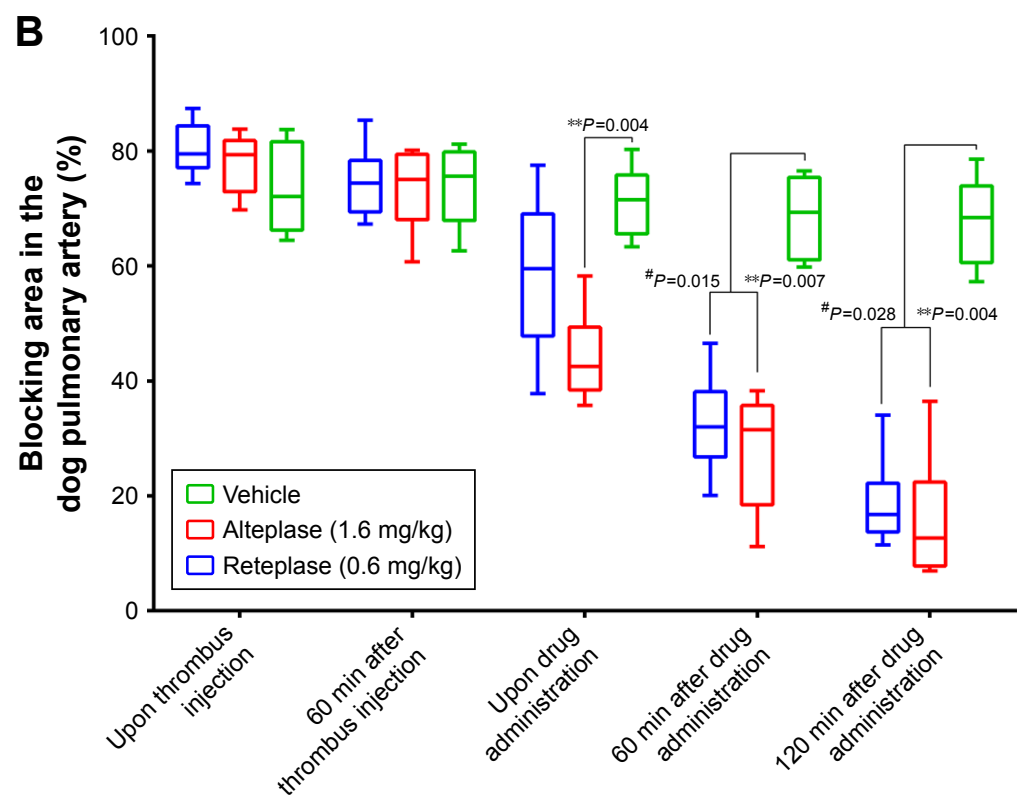

Time

Figure I Pulmonary angiography and blocking area in dog pulmonary artery.

Notes: (A) Pulmonary angiography of the embolic site in the pulmonary artery monitored by digital subtraction angiography. (B) The blocking area in the dog pulmonary artery in the reteplase group $\left({ }^{*} P<0.05, \mathrm{n}=6\right)$ and alteplase group $(* * P<0.01, n=6)$ was significantly decreased compared to the vehicle group after administration. Reperfusion rate of the pulmonary artery had no significant difference between the reteplase group and the alteplase group $(P>0.05, n=6)$. Data are presented as medians (range: minimum-maximum).

\section{Remission of pulmonary hemodynamic indexes by thrombolytic reteplase}

Abnormally elevated blood pressures caused by PTE may injure blood vessels. Therefore, the pulmonary arterial pressure, as a key indirect index for the evaluation of embolism, was monitored during the whole experimental process. Blockage caused by the injection of autologous thrombi led to increased resistance in the pulmonary artery. Consequently, pulmonary arterial systolic blood pressure (SBP), diastolic blood pressure (DBP), and MAP of dogs in the vehicle 
group (44.5 [34-57], 22.5 [14-33], and 29.5 [19-38] mmHg, respectively), the alteplase group (51 [40-61], 19 [16-24], and 27.5 [25-39] $\mathrm{mmHg}$, respectively), and the reteplase group (48.5 [33-58], 25.5 [17-34], and 33 [26-39] mmHg, respectively) increased dramatically upon thrombus injection and stabilized within 60 minutes (Figure $2 \mathrm{~A}-\mathrm{C}$ ). In contrast, dogs in the sham operation group exhibited consistently unchanged blood pressures during the experimental progress, with an SBP, DBP, and MAP of 27.5 (21-37), 11 (8.5-13), and $18.5(15-22) \mathrm{mmHg}$, respectively, 60 minutes
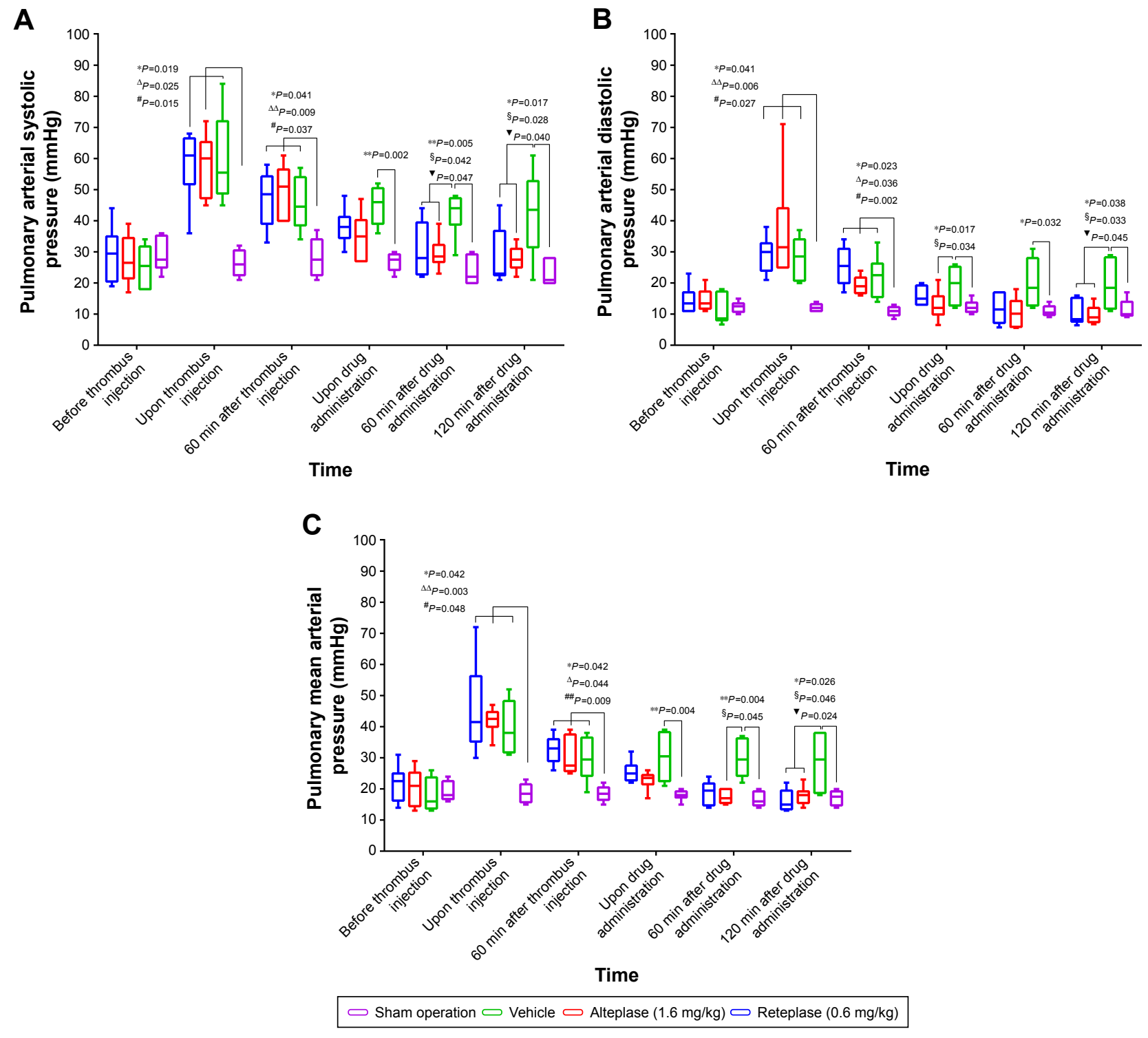

Figure 2 Reteplase dramatically reduced pulmonary hemodynamics after administration.

Notes: (A) Compared to the sham operation group, SBP of the pulmonary artery was increased significantly upon thrombus injection, and high SBP maintained through the experimental procedure $(* P<0.05, * * P<0.01, n=6)$. Upon thrombus injection and after thrombus injection for I hour, SBP was increased significantly in the reteplase group $\left({ }^{\#} P<0.05, n=6\right)$ and the alteplase group $\left({ }^{\Delta P}<0.05, \Delta P<0.0 \mathrm{I}, \mathrm{n}=6\right)$, compared with that of the sham operation group. One and two hours after drug administration, SBP of the reteplase group was downregulated nearly to the normal level, compared with the vehicle group $(\nabla P<0.05, n=6)$. Compared to the vehicle group, SBP was also remarkably decreased after administration of alteplase $(\$ P<0.05, n=6)$. Data are presented as medians (range: $\min -\max )$. (B) Compared to the sham operation group, $D B P$ of the pulmonary artery was increased significantly upon thrombus injection, and high DBP maintained through the experimental procedure $(* P<0.05$, $n=6)$. Upon thrombus injection and after thrombus injection for I hour, DBP was increased significantly in the reteplase group $\left({ }^{\#}<<0.05,{ }^{\#} P<0.0 \mathrm{I}, \mathrm{n}=6\right)$ and the alteplase group $\left({ }^{\triangle P}<0.05,{ }^{\triangle} P<0.0 \mathrm{I}\right.$, $\mathrm{n}=6$ ), compared with that of the sham operation group. Two hours after drug administration, DBP of the reteplase group $(\nabla P<0.05, n=6)$ and the alteplase group $(\$ P<0.05$, $\mathrm{n}=6$ ) was downregulated nearly to the normal level, compared with the vehicle group. Data are presented as medians (range: min-max). (C) Compared to the sham operation group, MAP of the pulmonary artery increased significantly upon thrombus injection, and high MAP was also maintained through the experimental procedure $(* P<0.05$, $* * P<0.01, n=6$ ). Upon thrombus injection and after thrombus injection for I hour, MAP was increased significantly in the reteplase group ( ${ }^{\#}<<0.05$, \# $P<0.0 \mathrm{I}, \mathrm{n}=6$ ) and the alteplase group $\left({ }^{\triangle P}<0.05, \Delta P<0.01, n=6\right)$, compared with that of the sham operation group. One and two hours after drug administration, MAP of the reteplase group $(\nabla P<0.05, n=6)$ and the alteplase group $(\$ P<0.05, n=6)$ was downregulated nearly to the normal level, compared with the vehicle group. Data are presented as medians (range: min-max). Time points in the experimental procedure were A, before thrombus injection; B, upon thrombus injection; C, I hour after thrombus injection; D, upon drug administration; E, I hour after drug administration; F, 2 hours after drug administration.

Abbreviations: DBP, diastolic blood pressure; MAP, mean arterial pressure; SBP, systolic blood pressure. 
after thrombus injection. Pulmonary SBP, DBP, and MAP detected in the vehicle group were consistently high from thrombus injection till 120 minutes after drug administration (SBP, DBP, and MAP: 43.5 [21-61], 18.5 [11-29], and 29.5 [18-38] mmHg, respectively). Whereas, pulmonary SBP, DBP, and MAP of animals in the reteplase and alteplase groups were all dramatically downregulated to a level similar to that of the sham operation group 120 minutes after drug administration (the reteplase group: 23 [21-45], 8.4 [6.4-16], and 16 [13-22] $\mathrm{mmHg}$; the alteplase group: 27.5 [22-34], 9 [6.8-15], and 18 [14-23] mmHg, respectively), significantly different from that of the vehicle group (Figure 2A-C). Our study indicated that one bolus dosing of reteplase $(0.6 \mathrm{mg} / \mathrm{kg})$ and infusion of alteplase $(1.6 \mathrm{mg} / \mathrm{kg}) \mathrm{had}$ no significant difference in the relief of pulmonary hypertension caused by autologous thrombus blockage. Consistent with the angiogram results, dramatic elevation of the pulmonary blood pressures suggested successful establishment of the acute PTE model, and the immediately decreased blood pressures upon reteplase administration verified its thrombolytic capability in this PTE study. ${ }^{21}$

\section{Impacts on blood coagulation}

Blood coagulation capability was evaluated during the thrombolytic process by measuring PT, APTT, and TT. Thrombus injection did not cause obvious change of PT, APTT, or TT in all groups. Administration of saline in both the sham operation and the vehicle group did not influence the blood coagulation capability. In contrast, animals in the reteplase and the alteplase group exhibited a dramatic extension of PT, APTT, and TT after drug administration, statistically different from the sham operation and the vehicle groups (Table 1). Besides, $0.6 \mathrm{mg} / \mathrm{kg}$ reteplase induced longer extension of PT, APTT, and TT than $1.6 \mathrm{mg} / \mathrm{kg}$ alteplase. Recovery of PT, APTT, and TT to the basal levels detected before thrombus injection was achieved on day 6 after drug administration in the reteplase and the alteplase groups (Table 1). No significant changes in FIB were detected at different time points in the sham group and the vehicle group. However, administration of both reteplase and alteplase significantly reduced the blood FIB level (Table 1). Moreover, animals in the reteplase group exhibited a consistent much lower FIB level $(0.25 \mathrm{~g} / \mathrm{L}$ in median) within 2 hours upon drug administration, compared to the alteplase group $(0.75 \mathrm{~g} / \mathrm{L}$ in median $)$.

\section{Impacts on hematological indexes}

Hematological indexes were all monitored during the experimental procedure: before thrombus injection, 60 minutes after thrombus injection, 60 minutes after drug administration, and 6 days after drug administration. None of the hematological indexes had any significant differences among the four groups before thrombus injection. White blood cell count, neutrophils, lymphocytes, eosinophils, basophils, and monocytes in the blood decreased slightly 60 minutes after thrombus injection, but increased 60 minutes after drug administration. No statistical difference was detected between any two groups on day 6 after administration of the thrombolytic agents. Platelet count in all four groups was lower compared to their basal levels after thrombus injection, and the decline ultimately recovered to the baseline on 6 day after drug administration. There was no obvious change in hematological indicators such as red blood cells, large un-staining cells, hemoglobin, hematocrit, mean corpuscular volume, mean corpuscular hemoglobin, mean corpuscular hemoglobin concentration, mean platelet volume, and reticulocytes throughout the whole experimental procedure (Table S1).

\section{Impacts on blood biochemical indexes}

Basic values of blood biochemical indexes were similar among all dogs in the four groups. After thrombus injection or drug treatment, these biochemical indexes changed in a similar manner in all groups, but no significant difference was observed among all groups ( $P>0.05$; Table $\mathrm{S} 2)$, which suggested no dysfunction of the kidney and liver during the study period.

\section{Impacts on blood gas indexes}

No remarkable changes of blood gas indexes such as $\mathrm{PCO}_{2}$, $\mathrm{pH}, \mathrm{HCO}_{3}$, and $\mathrm{TCO}_{2}$ were observed upon thrombus injection or drug administration in all four groups (Table $\mathrm{S} 3$ ).

\section{Results of anatomical observation and tissue pathological examination}

Our autopsy studies indicated that there was no obvious bleeding in all major organs. Thrombus organization was observed in four out of six dogs in the vehicle group by H\&E staining 6 days after drug administration, while no dog in the sham operation group exhibited these symptoms, suggesting successful establishment of the acute PTE model. No obvious thrombus organization was found in the pulmonary artery tissue $\mathrm{H} \& \mathrm{E}$ slices of the reteplase group and the alteplase group, indicating they could dissolve the thrombi and alleviate the occurrence of histopathologic symptoms. Nonspecific positive sites of Prussian blue staining were mainly observed in the blood cells of pulmonary artery, and no obvious positive staining was found in the lung tissue cells, indicating that no obvious bleeding occurred in the lung during reteplase thrombolysis (Figure 3). Consistent 


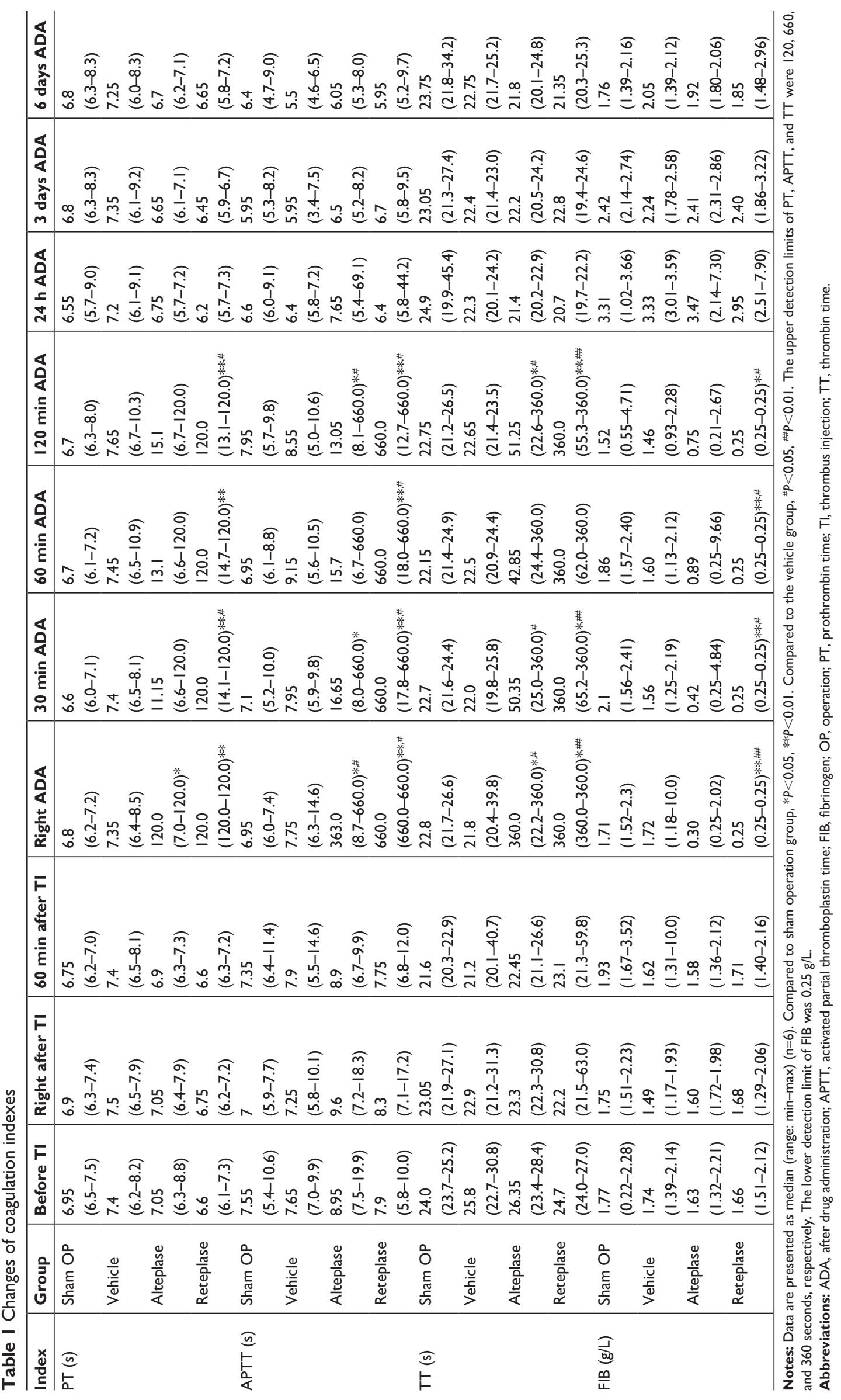


with previous biopsy studies of PTE patients, ${ }^{22}$ various degrees of granulomatous reactions were found in animals with thrombus injection. This might be attributed to inflammatory responses caused by fragments of the thrombus that blocked minute pulmonary vessels and lymphatics.

\section{Discussion}

Clinically, the gold standard for diagnosis of pulmonary embolism is angiography. ${ }^{23,24}$ Nearly $66 \%-93 \%$ of pulmonary embolisms could be detected by using pulmonary angiography or computed tomography. ${ }^{24,25}$ Previously animal studies of PTE mainly used blood pressure as the principle index. ${ }^{15,18,21}$ Here, we used pulmonary angiography in animal studies by innovative DSA, which provided real-time visualization all through, including accurate thrombus location, size and phenotypes; the conditions of arteries; and the thrombolysis procedure. Application of the minimally invasive interventional treatment enabled injection of the autologous thrombi specifically into the main pulmonary artery in the inferior lobe of the left lung, which endowed the study with high sensitivity, specificity, and replicability.

Mammalian PTE models including mice, rats, and piglets have been previously well established and studied. ${ }^{16,19,26-28}$ Compared to them, our canine system has several advantages. Firstly, living in a similar environment endows dogs and humans similar disease causes and developing processes, especially complex ones such as PTE and DVT. ${ }^{29}$ Since canines exhibit more genetic homogeneity with humans compared to other mammals, ${ }^{18,29}$ the analogous coagulation and fibrinolytic system might enhance the research accuracy and comparability, ${ }^{18,30,31}$ which would likely provide better clinical applications. Thrombolytic effects of alteplase on canine autologous thrombus were previously validated, ${ }^{32}$ and it was frequently used as a positive control in the canine coronary thrombosis model for efficacy evaluation of newly developed drugs. ${ }^{15,33}$ Alteplase $(1.5 \mathrm{mg} / \mathrm{kg}$ ) exhibited a much better thrombolytic effect than heparin $(10 \mathrm{U} / \mathrm{kg})$ in autologous canine PTE studies. ${ }^{34}$ Previous studies of a Beagle dog STEMI model found that canine autologous thrombus was composed of a large number of compacted FIB under a scanning electron microscope. Five of the six dogs exhibited coronary recanalization 2 hours after alteplase administration. ${ }^{32}$ Although the role of reteplase in PTE has not been clarified earlier, intra-arterial reteplase has been proved to induce similar reperfusion in canine basilar artery thrombosis studies as intravenous alteplase, and reteplase has been characterized with a lower rate of intracerebral hemorrhage. ${ }^{35}$

Secondly, commonly used IVC stenosis and ligation mouse models have obvious disadvantages, such as significant thrombus size variations, even absence of thrombus, and lack of blood flow recovery, which were avoided in our system. ${ }^{16}$ Usage of autologous thrombus largely excluded the occurrence of possible immunological reactions and activation of the canine fibrinolytic system. ${ }^{17,27}$ In order to better mimic disease occurrence, we optimized our thrombus preparation process. Previous studies usually used 3-8 hours for agglutination of canine autologous thrombus..$^{18,32,34}$ In one study, it was observed that a thrombus incubated in a $37^{\circ} \mathrm{C}$ water bath for 4 hours

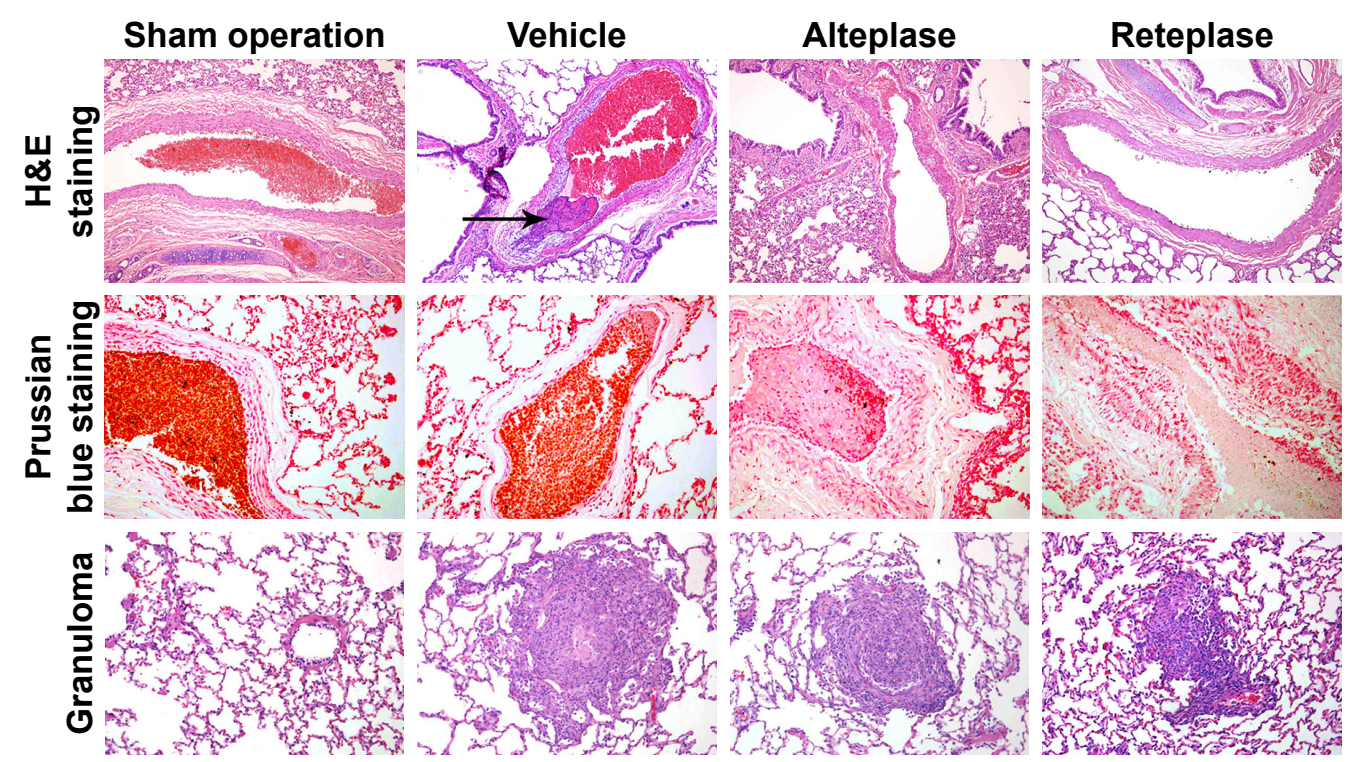

Figure 3 Thrombus formation, bleeding examination and inflammatory reactions in dog lung tissue.

Notes: Shown at $\times 200$ original magnification. Obvious thrombus formation and organization was shown in the paraffin section of the vehicle group stained by H\&E. No specific positive Prussian blue staining sites were observed in the pathological paraffin sections of different treatment groups. Different extents of granuloma symptom appeared in the thrombus-injected groups. The arrow points to thrombus formation in pulmonary artery. 
induced PTE with $\sim 65 \%$ increase of mean pulmonary artery pressure (mPAP), from 15.60 to $25.74 \mathrm{mmHg} .{ }^{34}$ In this study, the thrombus incubated in a $37^{\circ} \mathrm{C}$ water bath for 30 minutes, followed by $2^{\circ} \mathrm{C}-8^{\circ} \mathrm{C}$ overnight $(\sim 12-16$ hours $)$ induced a $137.5 \%$ increase of mPAP, from 16 to $38 \mathrm{mmHg}$. Hence, our thrombus might be endowed with better stability and blocking ability, which ensured maintenance of the PTE model and accurate drug evaluations. Lastly, in contrast to the difficulties of hemodynamic monitoring in mice, we monitored changes of blood pressures all through. In all, previous studies have adequately demonstrated the dissolving capability of canine autologous thrombus of both reteplase and alteplase. Hence, our studies well mimicked fatal PTE development in clinics; therefore, application of this animal model in preclinical studies of novel drugs would be of great clinical significance.

Alteplase has been approved by the US Food and Drug Administration (FDA) as clinical treatment for STEMI, acute stroke, and acute pulmonary embolism with different prescription dosages. For acute ischemic stroke patients, $0.9 \mathrm{mg} / \mathrm{kg}$ alteplase by intravenous injection was routinely administered 3 hours within stroke onset. ${ }^{36,37}$ The recommended dosage of alteplase was $100 \mathrm{mg}$ intravenous infusion in 2 hours for acute STEMI and pulmonary embolism. ${ }^{38,39}$ However, clinical acute pulmonary embolism meta-analysis studies indicated that $50 \mathrm{mg}$ alteplase by intravenous infusion in 2 hours was as effective as the standard $100 \mathrm{mg}$, and the lower dosage improved therapy safety with reduced bleeding risks. ${ }^{40-42}$ Previous canine acute PTE studies with autologous thrombosis indicated that $1.5 \mathrm{mg} / \mathrm{kg}$ alteplase was capable of dissolving the thrombi and recovering pulmonary pressures. ${ }^{34}$ Taken together, $1.6 \mathrm{mg} / \mathrm{kg}$ alteplase for dogs (equivalent to $50 \mathrm{mg} / 60 \mathrm{~kg}$ for humans) was used in our experiment, in order to achieve an obvious thrombolytic effect as positive control while avoiding bleeding side effects.

Reteplase was approved by the China FDA as a thrombolytic treatment of acute myocardial infarction in 2008 . As the third generation of thrombolytic agents, reteplase showed thrombolytic efficacy as treatment for STEMI/AMI patients, with a recommended regimen of $10 \mathrm{U}(18 \mathrm{mg})$ bolus dose, twice, 2 minutes each time with a 30-minute interval. ${ }^{13,43,44}$ However, up to now reteplase has not been approved as therapy for PTE. Therefore, it is necessary to evaluate its thrombolytic effects in preclinical studies first. Here, we developed a canine acute PTE model that showed clinical-like features, and used alteplase as a positive control, which was approved as treatment for acute pulmonary embolism and acute stroke in 2002 by the FDA. ${ }^{8}$ In order to achieve a parallel comparison with alteplase, we used half of the clinical dosage, one bolus of $0.6 \mathrm{mg} / \mathrm{kg}$ reteplase in dogs, equivalent to one dose of $10 \mathrm{U}(18 \mathrm{mg} / 60 \mathrm{~kg})$ for humans.

Consistent with previous studies, ${ }^{38,45}$ our studies indicated that pulmonary SBP, DBP, and MAP were elevated and sustained upon thrombus injection, and decreased after administration of thrombolytic agents, similar to successful performance in clinical therapies. The increased blood pressures may be attributed to the increased resistance in the pulmonary artery caused by thrombus blockage, and the large amount of endothelium-derived contracting factors released from the vascular endothelial cells and blood platelets. ${ }^{28,46}$ Meanwhile, the unchanged hemodynamic parameters in the sham operation group indicating the operation of surgery did not affect the blood pressures. Our angiography study vividly exhibited the process of thrombus formation, stabilization, dissolution, blockage, and recovery of blood flow, which were all consistent with changes of the hemodynamic parameters monitored. In all, thrombolytic effects of reteplase were vividly proved both directly and indirectly in our studies.

Administration of reteplase also led to dramatic changes in blood coagulation. While no obvious changes of coagulation parameters were observed in the vehicle and the sham operation group, PT, APTT, and TT were dramatically extended in groups with the thrombolytic regimens: reteplase or alteplase, and stronger effects were demonstrated by reteplase. Compared to the vehicle group and the sham operation group, both reteplase and alteplase led to decreased FIB levels, and the FIB level in the reteplase group was almost undetectable. These findings suggest that the thrombolytic effects of reteplase were attributed to its influence on the blood coagulation system, and reteplase demonstrated a better performance than alteplase. Recovery to the level before injection in both groups 24 hours after the operation suggested that thrombolytic drugs only had a temporary, rather than long-term, influence on blood coagulation, similar to studies of alteplase in other canine femoral artery thrombus models. ${ }^{47}$

As a most commonly used thrombolytic therapy, t-PAs such as alteplase share a similar side effect, dose-dependent risk of hemorrhagic complications. ${ }^{48,49}$ Due to the high incidence of bleeding, patients receiving thrombolytic treatments for acute pulmonary embolism required monitoring of specific nursing and care based on their clinical status. ${ }^{7,50}$ According to large randomized clinical trials of STEMI, the incidence, severity, and sites of bleeding events associated with reteplase treatment appeared to be similar to those associated with other fibrin-specific thrombolytic agents. ${ }^{13}$ In our study, no obvious bleeding was observed in the puncture/ catheter drug-administered sites and no cases of abnormal bleeding or death were found in the vehicle and the sham 
operation groups. These findings indicate that the disease animal model itself, the operation and the thrombus injection, would not cause the occurrence of bleeding. Therefore, our experimental system was suitable for evaluation of the potential bleeding side effects of novel thrombolytic therapies. With one bolus dosing of reteplase $(0.6 \mathrm{mg} / \mathrm{kg})$ in this study, only equivalent to half of the clinical dose in STEMI, no noticeable bleeding was observed in the Prussian blue staining of main organs such as lungs, while this dosage of reteplase was able to dissolve most thrombi. Therefore, we have reason to believe that this thrombolytic strategy not only guarantees thrombolytic effects, but also exhibits safety with less bleeding side effects.

Advantages of reteplase were demonstrated in preclinical studies as treatment for coronary artery or femoral artery thrombosis. ${ }^{51,52}$ Treatment of intermediate-risk acute pulmonary embolism with reteplase was effective and safe with no obvious side effects. ${ }^{53}$ Reteplase exhibited a longer activity half-life (12.6 minutes) than alteplase ( $<5$ minutes) in patients with acute myocardial infarction, ${ }^{13}$ and less platelet aggregation was observed in the early phase of acute myocardial infarction patients treated by reteplase in contrast to alteplase.$^{54}$ Clinical studies of massive pulmonary embolism indicated that reteplase led to a quick release of pulmonary resistance within 0.5 hours, in contrast to 2 hours in the alteplase group. ${ }^{38}$ In our study, the pulmonary DSA angiography results indicated that one single bolus dosing of reteplase $(0.6 \mathrm{mg} / \mathrm{kg})$ initiated embolism relief within seconds, and led to almost $90 \%$ pulmonary artery reperfusion 2 hours after drug administration. What is more, half of the clinical effective dosage of reteplase $(0.6 \mathrm{mg} / \mathrm{kg})$ exerted an efficacy comparable to the clinically proved effective dosage of alteplase $(1.6 \mathrm{mg} / \mathrm{kg})$. The efficiency of reteplase might be attributed to its longer half-life or better affinity to plasminogen, which needs further studies for clarification. In addition, intravenous injection of reteplase is simple and easy, which may avoid drug treatment errors under emergency circumstances. Taken together, reteplase may be characterized as a safer, quicker, and more effective thrombolytic therapy for PTE patients than alteplase. ${ }^{13}$

In all, our novel canine acute PTE model was well characterized by resembling major clinical features, such as blocked pulmonary vessels and increased pulmonary arterial pressures. Reteplase, which dissolved the thrombus quickly and efficiently, exhibited a remarkable therapeutic effect on this acute PTE model. Our findings indicated the potential of reteplase in the clinical treatment of acute PTE. This system may greatly contribute to the design and evaluation of new prophylaxis, diagnostic approaches, or therapeutic treatments against PTE. In addition, this technically innovative, stability- and validity-proved model provided a unique human-like biological environment, in which the biology behind relevant diseases, such as pulmonary embolism, DVT, cerebral infarction, ischemic myocardial infarction, hypertension, and heart attack, could also be investigated.

\section{Conclusion}

Our preclinical study has proved for the first time that reteplase is an effective and safe therapy for PTE, and a small dosage of reteplase exerts an efficacy comparable to the routine dosage of alteplase. Our findings indicated the potential of reteplase as a clinical treatment against PTE. We developed a novel canine acute PTE model characterized by a minimally invasive method and DSA, which made "what you see is what you get" of PTE progression possible. This experimental system could have great applications in the screening and evaluation of new anti-PTE or relevant prophylactic, therapeutic, and diagnostic approaches.

\section{Acknowledgment}

The research leading to these results has received funding from the National Natural Scientific Foundation of China (81571177; 81771310).

\section{Disclosure}

Yinbing Zhang, Yanyan Zhang, Jie Zhang, Xin Wang, and Zhihui Zhong are employed by Sichuan Kangcheng Biomed Co., Ltd., Chengdu, China. Haifeng Liu and Xiangshan Zhou are employed by Angde Biotech Pharmaceutical Co., Ltd., Liaocheng, China. The authors report no other conflicts of interest in this work.

\section{References}

1. Friedman T, Winokur RS, Quencer KB, Madoff DC. Patient Assessment: Clinical Presentation, Imaging Diagnosis, Risk Stratification, and the Role of Pulmonary Embolism Response Team. Semin Intervent Radiol. 2018;35(2):116-121.

2. Konstantinides SV, Barco S, Lankeit M, Meyer G. Management of Pulmonary Embolism: An Update. J Am Coll Cardiol. 2016;67(8):976-990.

3. Heit JA. The epidemiology of venous thromboembolism in the community. Arterioscler Thromb Vasc Biol. 2008;28(3):370-372.

4. Rahimtoola A, Bergin JD. Acute pulmonary embolism: an update on diagnosis and management. Curr Probl Cardiol. 2005;30(2):61-114.

5. Chatterjee S, Chakraborty A, Weinberg I, et al. Thrombolysis for pulmonary embolism and risk of all-cause mortality, major bleeding, and intracranial hemorrhage: a meta-analysis. JAMA. 2014;311(23) 2414-2421.

6. Wiener RS, Schwartz LM, Woloshin S. Time trends in pulmonary embolism in the United States: evidence of overdiagnosis. Arch Intern Med. 2011;171(9):831-837.

7. Smithburger PL, Campbell S, Kane-Gill SL. Alteplase treatment of acute pulmonary embolism in the intensive care unit. Crit Care Nurse. 2013;33(2):17-27.

8. Ouellette DW, Patocka C. Pulmonary embolism. Emerg Med Clin North Am. 2012;30(2):329-375. 
9. Kearon C, Akl EA, Comerota AJ, et al. Antithrombotic therapy for VTE disease: Antithrombotic Therapy and Prevention of Thrombosis, 9th ed: American College of Chest Physicians Evidence-Based Clinical Practice Guidelines. Chest. 2012;141(2 Suppl):e419S-e494S.

10. Jaff MR, Mcmurtry MS, Archer SL, et al. Management of massive and submassive pulmonary embolism, iliofemoral deep vein thrombosis, and chronic thromboembolic pulmonary hypertension: a scientific statement from the American Heart Association. Circulation. 2011; 123(16):1788-1830.

11. Thabut G, Thabut D, Myers RP, et al. Thrombolytic therapy of pulmonary embolism: a meta-analysis. J Am Coll Cardiol. 2002;40(9): 1660-1667.

12. Goldhaber SZ, Visani L, de Rosa M. Acute pulmonary embolism: clinical outcomes in the International Cooperative Pulmonary Embolism Registry (ICOPER). Lancet. 1999;353(9162):1386-1389.

13. Simpson D, Siddiqui MA, Scott LJ, Hilleman DE. Spotlight on reteplase in thrombotic occlusive disorders. BioDrugs. 2007;21(1):65-68.

14. Martin U, Kaufmann B, Neugebauer G. Current clinical use of reteplase for thrombolysis. A pharmacokinetic-pharmacodynamic perspective. Clin Pharmacokinet. 1999;36(4):265-276.

15. Martin U, Sponer G, Strein K. Evaluation of thrombolytic and systemic effects of the novel recombinant plasminogen activator BM 06.022 compared with alteplase, anistreplase, streptokinase and urokinase in a canine model of coronary artery thrombosis. J Am Coll Cardiol. 1992; 19(2):433-440.

16. Diaz JA, Obi AT, Myers DD, et al. Critical review of mouse models of venous thrombosis. Arterioscler Thromb Vasc Biol. 2012;32(3): $556-562$.

17. Kjaergaard B, Kristensen SR, Risom M, Larsson A. A porcine model of massive, totally occlusive, pulmonary embolism. Thromb Res. 2009; 124(2):226-229.

18. Deng C, Wu D, Zhai Z, et al. Close concordance between pulmonary angiography and pathology in a canine model with chronic pulmonary thromboembolism and pathological mechanisms after lung ischemia reperfusion injury. J Thromb Thrombolysis. 2016;41(4):581-591.

19. Roehl AB, Steendijk P, Baumert JH, Schnoor J, Rossaint R, Hein M. Comparison of 3 methods to induce acute pulmonary hypertension in pigs. Comp Med. 2009;59(3):280-286.

20. Miller GA, Sutton GC, Kerr IH, Gibson RV, Honey M. Comparison of streptokinase and heparin in treatment of isolated acute massive pulmonary embolism. Br Heart J. 1971;33(4):616.

21. Tagami H, Utoh J, Sun LB, et al. Effects of recombinant tissue-type plasminogen activator on life-threatening acute pulmonary thromboembolism in a canine model: a comparative study of e6010 and alteplase. Ann Thorac Cardiovasc Surg. 2000;6(5):299-303.

22. Baydur A, Koss MN, Sharma OP, et al. Microscopic pulmonary embolisation of an indwelling central venous catheter with granulomatous inflammatory response. Eur Respir J. 2005;26(2):351-353.

23. Schoepf UJ, Goldhaber SZ, Costello P. Spiral computed tomography for acute pulmonary embolism. Circulation. 2004;109(18):2160-2167.

24. Wolf SJ, Mccubbin TR, Nordenholz KE, Naviaux NW, Haukoos JS. Assessment of the pulmonary embolism rule-out criteria rule for evaluation of suspected pulmonary embolism in the emergency department. Am J Emerg Med. 2008;26(2):181-185.

25. Golpe R, de Llano LA, Castro-Añón O, Vázquez-Caruncho M, González-Juanatey C, Fariñas MC. Long-term outcome of patients with persistent vascular obstruction on computed tomography pulmonary angiography 6 months after acute pulmonary embolism. Acta Radiol. 2012;53(7):728-731.

26. Weissmann N, Sydykov A, Kalwa H, et al. Activation of TRPC6 channels is essential for lung ischaemia-reperfusion induced oedema in mice. Nat Commun. 2012;3:649.

27. Runyon MS, Gellar MA, Sanapareddy N, Kline JA, Watts JA. Development and comparison of a minimally-invasive model of autologous clot pulmonary embolism in Sprague-Dawley and Copenhagen rats. Thromb J. 2010;8:3.
28. Li CY, Deng W, Liao XQ, Deng J, Zhang YK, Wang DX. The effects and mechanism of ginsenoside Rg1 on myocardial remodeling in an animal model of chronic thromboembolic pulmonary hypertension. Eur J Med Res. 2013;18:16.

29. Shearin AL, Ostrander EA. Leading the way: canine models of genomics and disease. Dis Model Mech. 2010;3(1-2):27-34.

30. Fu J, Ren J, Zou L, Bian G, Li R, Lu Q. The thrombolytic effect of miniplasmin in a canine model of femoral artery thrombosis. Thromb Res. 2008;122(5):683-690.

31. Ben SQ, Ni SS, Shen HH, et al. The dynamic changes of LDH isoenzyme 3 and D-dimer following pulmonary thromboembolism in canine. Thromb Res. 2007;120(4):575-583.

32. Zhang H, Cui YC, Tian Y, et al. A novel model for evaluating thrombolytic therapy in dogs with ST-elevation myocardial infarction. BMC Cardiovasc Disord. 2016;16:21.

33. Wang K, Zhou X, Huang Y, et al. Adjunctive treatment with ticagrelor, but not clopidogrel, added to tPA enables sustained coronary artery recanalisation with recovery of myocardium perfusion in a canine coronary thrombosis model. Thromb Haemost. 2010;104(3):609-617.

34. Wang Y, Wang C, Yang YY, Pang PB. Effect of recombinant singlechain urokinase-type plasminogen activator on experimental pulmonary embolism. Clin Appl Thromb Hemost. 2010;16(5):537-542.

35. Qureshi AI, Boulos AS, Hanel RA, et al. Randomized comparison of intra-arterial and intravenous thrombolysis in a canine model of acute basilar artery thrombosis. Neuroradiology. 2004;46(12):988-995.

36. Hacke W, Kaste M, Fieschi C, et al. Randomised double-blind placebo-controlled trial of thrombolytic therapy with intravenous alteplase in acute ischaemic stroke (ECASS II). Second EuropeanAustralasian Acute Stroke Study Investigators. Lancet. 1998;352(9136): $1245-1251$.

37. Clark WM, Albers GW, Madden KP, Hamilton S. The rtPA (alteplase) 0- to 6-hour acute stroke trial, part A (A0276g): results of a double-blind, placebo-controlled, multicenter study. Thromblytic therapy in acute ischemic stroke study investigators. Stroke. 2000;31(4):811-816.

38. Tebbe U, Graf A, Kamke W, et al. Hemodynamic effects of double bolus reteplase versus alteplase infusion in massive pulmonary embolism. Am Heart J. 1999;138(1 Pt 1):39-44.

39. Martínez-Ríos MA, Rosas M, González H, et al. Comparison of reperfusion regimens with or without tirofiban in ST-elevation acute myocardial infarction. Am J Cardiol. 2004;93(3):280-287.

40. Zhang Z, Zhai ZG, Liang LR, Liu FF, Yang YH, Wang C. Lower dosage of recombinant tissue-type plasminogen activator (rt-PA) in the treatment of acute pulmonary embolism: a systematic review and meta-analysis. Thromb Res. 2014;133(3):357-363.

41. Wang C, Zhai Z, Yang Y, et al. Efficacy and safety of low dose recombinant tissue-type plasminogen activator for the treatment of acute pulmonary thromboembolism: a randomized, multicenter, controlled trial. Chest. 2010;137(2):254-262.

42. Brandt K, Mcginn K, Quedado J. Low-Dose Systemic Alteplase (tPA) for the Treatment of Pulmonary Embolism. Ann Pharmacother. 2015;49(7):818-824.

43. Jinatongthai P, Kongwatcharapong J, Foo CY, et al. Comparative efficacy and safety of reperfusion therapy with fibrinolytic agents in patients with ST-segment elevation myocardial infarction: a systematic review and network meta-analysis. Lancet. 2017;390(10096):747-759.

44. Hoffmeister HM, Kastner C, Szabo S, et al. Fibrin specificity and procoagulant effect related to the kallikrein-contact phase system and to plasmin generation with double-bolus reteplase and front-loaded alteplase thrombolysis in acute myocardial infarction. Am J Cardiol. 2000;86(3):263-268.

45. Cau SB, Dias-Junior CA, Montenegro MF, de Nucci G, Antunes E, Tanus-Santos JE. Dose-dependent beneficial hemodynamic effects of BAY 41-2272 in a canine model of acute pulmonary thromboembolism. Eur J Pharmacol. 2008;581(1-2):132-137.

46. Jorens PG, van Marck E, Snoeckx A, Parizel PM. Nonthrombotic pulmonary embolism. Eur Respir J. 2009;34(2):452-474. 
47. Suzuki N, Suzuki S, Nagaoka N, et al. Thrombolysis of canine femoral artery thrombus by a novel modified tissue-type plasminogen activator (E6010). Jpn J Pharmacol. 1994;65(3):257-263.

48. Chamsuddin A, Nazzal L, Kang B, et al. Catheter-directed thrombolysis with the Endowave system in the treatment of acute massive pulmonary embolism: a retrospective multicenter case series. J Vasc Interv Radiol. 2008;19(3):372-376.

49. Dong BR, Hao Q, Yue J, Wu T, Liu GJ. Thrombolytic therapy for pulmonary embolism. Cochrane Database Syst Rev. 2009;3:CD004437.

50. Wan S, Quinlan DJ, Agnelli G, Eikelboom JW. Thrombolysis compared with heparin for the initial treatment of pulmonary embolism: a meta-analysis of the randomized controlled trials. Circulation. 2004; 110(6):744-749.

51. Martin U, Kohnert U, Stern A, Popp F, Fischer S. Comparison of the recombinant Escherichia coli-produced protease domain of tissue-type plasminogen activator with alteplase, reteplase and streptokinase in a canine model of coronary artery thrombolysis. Thromb Haemost. 1996; 76(6):1096-1101.
52. Nakada MT, Montgomery MO, Nedelman MA, et al. Clot lysis in a primate model of peripheral arterial occlusive disease with use of systemic or intraarterial reteplase: addition of abciximab results in improved vessel reperfusion. J Vasc Interv Radiol. 2004;15(2 Pt 1):169-176.

53. Zhao HG, Wang SX, Lu ZN, Zn L, et al. Clinical efficacy and safety of thrombolytic treatment with reteplase in patients with intermediaterisk acute pulmonary embolism. Zhonghua Xin Xue Guan Bing Za Zhi. 2017;45(4):314-317.

54. Moser M, Nordt T, Peter K, et al. Platelet function during and after thrombolytic therapy for acute myocardial infarction with reteplase, alteplase, or streptokinase. Circulation. 1999;100(18):1858-1864. 


\section{Supplementary materials}

Video S1 Representative video of pulmonary embolism in Beagle dogs with digital subtraction angiography one hour after treatment: the normal pulmonary artery flow in the sham operation group.

Video S2 Representative video of pulmonary embolism in Beagle dogs with digital subtraction angiography one hour after treatment: massive embolism was still checked in pulmonary artery of the vehicle group.

Video S3 Representative video of pulmonary embolism in Beagle dogs with digital subtraction angiography one hour after treatment: pulmonary artery thrombosis was clearly dissolved and the reperfusion of blood flow was very obvious after infusion of alteplase $(1.6 \mathrm{mg} / \mathrm{kg})$.
Video S4 Representative video of pulmonary embolism in Beagle dogs with digital subtraction angiography one hour after treatment: the thrombolytic effect of one bolus administration of reteplase $(0.6 \mathrm{mg} / \mathrm{kg})$ was very significant under digital subtraction angiography.

\section{Publish your work in this journal}

Drug Design, Development and Therapy is an international, peerreviewed open-access journal that spans the spectrum of drug design and development through to clinical applications. Clinical outcomes, patient safety, and programs for the development and effective, safe, and sustained use of medicines are the features of the journal, which has also been accepted for indexing on PubMed Central. The manuscript management system is completely online and includes a very quick and fair peer-review system, which is all easy to use. Visit http://www.dovepress.com/testimonials.php to read real quotes from published authors. 